\title{
Das Associationsgesetz der unendlichen Reihen und Producte.
}

\author{
Von F. Hočevar in Brünn.
}

Leitet man aus einer gegebenen unendlichen Reihe eine zweite $a b$, indem man die Glieder der ersten ohne Änderungder Aufeinanderfolge gruppenweise zu neuen Gliedern vereinigt, so liegen die Unbestimmtheitsgrenzen der zweiten Reihe zwischen jenen der ersten oder sie fallen mit denselben zur sammen. Ein analoger Satz gilt auch für unendliche Producte. Diese beiden Sätze drücken das Verhalten der unendlichen Reihen und Producte bei associativen Umformungen in der einfachsten und zugleich allgemeinsten Weise aus und können daher als das Associationsgesetz der unendlichen Reihen und Producte bezeichnet werden.

1. Zur Ableitung dieses Gesetzes soll zunächst folgender Hilfssatz bewiesen werden:

Sind die Werte einer Größe $c_{n}$ für alle positiven ganzen $n$ gegeben, und lässt man $n$ einmal die Reihe der natürlichen $Z$ ahlen und ein anderesmal irgend eine andere Reihe positiver ganzer Zahlen $m_{1}, m_{2}, m_{3}, \ldots$ durchlaufen, wobei $m_{1}<m_{2}<m_{3}<\ldots$ vorausgesetzt wird, so liegen für lim $n=\infty$ die Unbestimmtheitsgrenzen von $c_{m_{n}}$ zwischen jenen von $c_{n}$ oder sie fallen mit denselben zusammen.

Bezeichnet man, um diesen Satz zu beweisen, mit $G(x)$ die obere und mit $K(x)$ die untere Grenze aller dem Intervall $(x, \infty)$ angehörigen Glieder der Reihe

$$
c_{1}, c_{2}, c_{3}, \ldots
$$

so haben diese Functionen, weil sie monoton verlaufen, für $\lim x=\infty$ bestimmte Grenzwerte, etwa 0 und $U$, welche die obere, bezw. untere Unbestimmtheitsgrenze von $c_{n}$ für $\lim n=\infty$ heifen. Wir setzen also

$$
\lim G(x)=0, \lim K(x)=U, \text { für } \lim x=\infty,
$$


bemerken jedoch, dass sich diese Definitionsgleichungen von den folgenden

$$
\lim G\left(c_{n}\right)=0, \lim K\left(c_{n}\right)=U \text { für } \lim n=\infty,
$$

nicht wesentlich unterscheiden. ${ }^{1}$ )

Nun greifen wir aus der Reihe (1) unendlich viele Zahlen

$$
c_{m_{1},}, c_{m_{2}}, c_{m_{3}}, \ldots
$$

heraus, ohne ihre ursprüngliche Stellung gegen einander zu verändern, (d. h. es sei $m_{1}<m_{2}<m_{3}<\ldots$ ) und bezeichnen analog mit $G_{1}(x)$ die obere und mit $K_{1}(x)$ die untere Grenze der in das Intervall $(x, \infty)$ fallenden Glieder der Reibe (2). Es existieren dann gewisse Grenzwerte

$$
\lim G_{1}(x)=O_{1}, \lim K_{1}(x)=U_{1} \text { für } \lim x=\infty,
$$

welche die obere, bezw. "untere Unbestimmtheitsgrenze von $c_{m_{n n}}$ für $\lim n=\infty$ heißen sollen.

Aus dem Begriffe der oberen und der unteren Grenze einer Zahlenreihe folgt num

$$
G(x) \geq G_{1}(x) \geq K_{1}(x) \geq K(x),
$$

weil nämlich alle dem Intervall $(x, \infty)$ angehörenden Glieder der Reihe (2) zugleich unter den demselben Intervall angehörenden Gliedern der Reihe (1) vorkommen und weil selbstverständlich $G_{1}(x) \geq K_{1}(x)$ sein muss. Hieraus ergibt sich durch den Grenzübergang $\lim x=\infty$

$$
0 \geq O_{1} \geq U_{1} \geq U
$$

womit der oben ausgesprochene Hilfssatz bewiesen ist.

Man ersieht aus diesem Satze, dass für $O=U$ anch $O_{1}=U_{1}$ ist.

Existiert also der Grenzwert $\lim c_{n}$ für $\lim n=\infty$, so existiert auch der Grenzwert $\lim c_{m_{n}}$ für $\lim n=\infty$ und zwar sind die beiden Grenzwerte gleich.

2. Um nun die Eingangs erwähnten Sätze zu beweisen, leiten wir aus der unendlichen Reihe

$$
a_{1}+a_{2}+a_{3}+\ldots
$$

1) Vgl. den Aufsatz des Verfassers: „Über die Convergenz bestimmter Integrale mit unendlichen Grenzen" im IV. Jahrgang dieser Monatshefte, p. 181. 
die Reihe

$$
A_{1}+A_{2}+A_{3}+\ldots
$$

ab, indem wir die Glieder der ersten entsprechend den Gleichungen

$$
\begin{gathered}
a_{1}+a_{2}+\ldots+a_{m_{1}}=A_{1}, \\
a_{m_{1}+1}+a_{m_{1}+2}+\ldots+a_{m_{2}}=A_{2},
\end{gathered}
$$

zu Gliedern der zweiten vereinigen. Ist also $s_{n}$ die Summe der ersten $n$ Glieder der Reihe (3), so ist $s_{m_{n}}$ die Summe der ersten $n$ Glieder der Reihe (4). Da ferner die Unbestimmtheitsgrenzen von $s_{n}$ und $s_{m n_{n}}$ für $\lim n=\infty$ als die Unbestimmtheitsgrenzen der entsprechenden Reihen (3) und (4) bezeichnet werden, so erkennt man mittelst des unter 1 . bewiesenen Hilfssatzes ohneweiters, dass. die Unbestimmtheitsgrenzen der Reihe (4) zwischen jenen der Reihe (3) liegen oder mit denselben zusammenfallen.

Hieraus folgt als specieller Fall der bekannte Satz, dass man aus einer convergenten Reihe wieder eine convergente Reihe mit derselben Summe erhält, wenn man die Glieder der ersten ohne Änderung der Aufeinanderfolge gruppenweise zu Gliedern der zweiten vereinigt.

Man kann auch von der Reihe (4) ausgehen und die Glieder derselben durch gleichwertige Aggregate von Zahlen ersetzen, welche dann als die Glieder der abgeleiteten Reihe (3) zu betrachten sind. Es ergibt sich aus dem Vorausgehenden, dass dadurch die Unbestimmtheitsgrenzen der unendlichen Reihe auseinander gerückt werden oder unverändert bleiben.

Aus der Convergenz der Reihe (4) kann daher, wie bekannt, im allgemeinen nicht auf die Convergenz der Reihe (3) geschlossen werden.

Auf ähnlichem Wege lässt sich beweisen, dass die Unbestimmtheitsgrenzen des unendlichen Productes

$$
B_{1} B_{2} B_{3} \ldots
$$

zwischen jenen des unendlichen Productes

$$
b_{1} b_{2} b_{3} \ldots
$$

liegen oder mit denselben zusammenfallen, wenn zwischen den Factoren der beiden Producte die Beziehungen

$$
\begin{gathered}
B_{1}=b_{1} b_{2} \ldots b_{m_{1}}, \\
B_{2}=b_{m_{1}+1} b_{m_{1}+2} \ldots b_{m_{2}},
\end{gathered}
$$


bestehen. Man orkennt die Richtigkeit dieser Behauptung, wenn man

daher

$$
b_{1} \quad b_{2} \ldots b_{n}=p_{n}
$$

$$
B_{1} B_{2} \ldots B_{n}=p_{i n_{n}}
$$

setzt und hierauf den in 1 . bewiesenen Hilfssatz anwendet.

Hieraus ergibt sich der bekannte Satz, dass aus der Convergenz des Productes (6) jene des Productes (5) tolgt, während die Umkehrung nicht allgemein zulässig ist.

3. Für das praktische Rechnen sind namentlich solche associative Transformationen von Wichtigkeit, welche die Unbestimmtheitsgrenzen einer beliebigen (convergenten oder divergenten) unendlichen Reihe oder eines beliebigen unendlichen Productes unverändert lassen. Eine solche Umformung ergibt sich aus dem unter 1. abgeleiteten Hilfssatze, wenn man die Zahlen

$$
c_{m \eta_{1}}, c_{m m_{2}}, c_{m m_{3}}, \ldots
$$

so wählt, dass sich $c_{n}$ bei wachsendem $n$ in allen von je zwei aufeinander folgenden Zahlen der Reihe (7) begrenzten Intervallen monoton verändert. Dann ist für beliebige $n$ die eine der beiden Zablen $c_{m_{n}}$ und $c_{m_{n+1}}$ die obere und die andere die untere Grenze aller in der ursprünglichen Anordnung zwischen diesen beiden Zablen vorkommenden Glieder der Reihe $c_{1}, c_{2}, c_{3}, \ldots$ Lässt man also alle diese Zahlen mit Ausnahme der in (7) angeführten weg, so werden dadurch die oberen Grenzen $G\left(c_{m_{1}}\right), G\left(c_{m_{2}}\right), \ldots$ und die unteren Grenzen $K\left(c_{m_{1}}\right), K\left(c_{m_{2}}, \ldots\right.$ nicht geändert. Daraus folgt

$$
G(x)=G_{1}(x) \text { and } K(x)=K_{1}(x)
$$

für $x=c_{m_{1}}, c_{m_{2}}, \ldots$ und daher auch

$$
O=O_{1} \text { and } U=U_{1} \text {. }
$$

Die hier bezüglich $c_{n}$ gemachte Voraussetzung wird von der Reihensumme $s_{n}$ erfüllt, wenn in jedem Gliede $A_{n}$ von einem bestimmbaren $n$ angefangen nur gleichbezeichnete Glieder $a_{n}$ vereinigt sind. Man erhält somit den Satz:

Die Unbestimmtheitsgrenzen einer unendlichen Reihe werden nicht geändert, wenn man von einem bestimmten Gliededer Reiheangefangen stets gleichbezeichnete aufeinander folgende Glieder zu neuen Gliedern vereinigt oder die Glieder der gegebenen Reibe in gleichbezeichnete neue Glieder zerlegt. 
Hieraus folgt u. A., dass sich jede unendliche Reihe, in welcher Gruppen von positiven Gliedern mit Gruppen von negativen ohne Ende abwechseln, in eine alternierende Reihe verwandeln lässt, ohne dass hiedurch die Unbestimmtheitsgrenzen der Reihe geändert werden.

Wenn z. B. die Reihe vorliegt, welche aus den reciproken Werten der natürlichen Zahlen derart gebildet ist, dass die ersten $m$ Werte mit positivem, die folgenden $2 m$ mit negativem, die darauffolgenden $3 m$ wieder mit positivem Vorzeichen $u$. s. f. genommen werden, so ist diese Reihe dann und nur dann convergent, wenn auch die Reihe

$$
A_{1}-A_{2}+A_{3}-A_{4}+\ldots
$$

wo

$$
A_{n}=\frac{1}{\left(\begin{array}{l}
n \\
2
\end{array}\right) m+1}+\frac{1}{\left(\begin{array}{l}
n \\
2
\end{array}\right) m+2}+\cdots+\frac{1}{\left(\begin{array}{c}
n+1 \\
2
\end{array}\right) m}
$$

bedeutet, convergiert. Dies ist wirklich der Fall, denn man hat

$$
A_{n+1}<A_{n} \text { und } \lim A_{n}=0 \text { für } \lim n=\infty \text {, }
$$

was mit Hilfe der Formel ${ }^{1}$ )

$$
A_{n}=l\left(1+\frac{m n}{\left(\begin{array}{l}
n \\
2
\end{array}\right) m+\vartheta}\right), 0<\vartheta<1,
$$

leicht bewiesen wird.

4. Man kann aus einer gegebenen Reihe mit positiven und negativen Gliedern durch associative Umformungen auch Reihen ableiten, welche dieselbe obere (untere) und im allgemeinen eine andere untere (obere) Unbestimmtheitsgrenze besitzen. Das Verfahren ergibt sich aus dem unter 1. abgeleiteten Hilfssatze, wenn man die Zahlen $c_{m_{1}}, c_{m_{2}}, c_{m_{3}}, \ldots$ so wählt, dass sämmtliche in der ursprünglichen Anordnung zwischen irgend zwei aufeinander folgenden dieser Zahlen, z. B. $c_{m_{n}}$ und $c_{m_{n}+1}$, vorkommenden Glieder der Reihe $c_{1}, c_{2}, c_{3}, \ldots$ nicht größer (kleiner) sind als die größere (kleinere) dieser beiden Zahlen. Es ist dann in dem einen Falle

$$
G(x)=G_{1}(x) \text { für } x=c_{m_{1}}, c_{m_{2}}, c_{m_{3}}, \ldots
$$

und daher auch $0=0_{1}$, hingegen im anderen

und daher auch $U=U_{1}$.

$$
K(x)=K_{1}(x) \text { für } x=c_{m_{1}}, c_{m_{2}}, c_{m_{3}}, \ldots
$$

1) Vgl. Schlömileh, Comp., I. Band, 3. Aufl, pag. 171. 
Die hier bezüglich $c_{n}$ gemachte Voraussetzung wird vom Summenglied $s_{n}$ einer unendlichen Reihe, in welcher Gruppen von positiven mit Gruppen von negativen Gliedern abwechseln, erfüllt, wenn man von einer bestimmten Stelle in der Reihe an jede negative (positive) Gruppe mit der nachfolgenden positiven (negativen) Gruppe zu einem neuen Gliede vereinigt. Bei diesem Verfahren bleibt die obere (untere) Unbestimmtheitsgrenze unverändert. So hat z. B. die Reihe

$$
a_{1}-a_{2}+a_{3}-a_{4}+\ldots
$$

dieselbe obere Unbestimmtheitsgrenze wie die Reihe

$$
a_{1}+\left(-a_{2}+a_{3}\right)+\left(-a_{4}+a_{5}\right)+\ldots
$$

and dieselbe untere Unbestimmtheitsgrenze wie die Reihe

$$
\left(a_{1}-a_{2}\right)+\left(a_{3}-a_{4}\right)+\left(a_{5}-a_{6}\right)+\ldots,
$$

wenn sämmtliche $\alpha$ positiv sind und die eingeklammerten Binome als Reihenglieder aufgefasst werden. Hieraus folgt u. A., dass die untere Unbestimmtheitsgrenze der Reihe (8) zugleich die obere der folgenden Reihe ist:

$$
-a_{2}+a_{1}-a_{3}+a_{4}-a_{5}+a_{6}-\ldots
$$

Schließlich sei noch bemerkt, dass sich die in den Artikeln 3 und 4 gewonnenen Resultate ohneweiters auf unendliche Producte übertragen lassen, wenn man den positiven (negatiren) Reihengliedern solche Factoren des unendlichen Productes entsprechen lässt, welche größer (kleiner) als 1 sind. 